\section{Particle Shape}

Mr. E. J. W. WhitTakeR has directed attention ${ }^{1}$ to the frequent use of the term 'needle-shaped' in chemical literature as a loose description of certain crystal shapes, and proposes a method of calculating an 'acicularity coefficient' to make this description. more precise. Such a coefficient, however, would be characteristic not of the substance, but only of a particular batch of it formed under conditions which give rise to the particular 'habit'.

The bare description, 'needle-shaped', occurs frequently when a complete crystallographic description seems desirable. Its use is sometimes a confession of failure, the crystals being so thin that the observer cannot discover the exact nature of the terminations. Sometimes one feels that the term merely expresses lack of interest in the crystallographic details.

The measurements which Mr. Whittaker suggests (three on each crystal) would involve a considerable amount of labour. If one's purpose is to deseribe a substance, it would seem better to expend the effort on an attempt to determine a few crystallographic constants which would be truly characteristic. If, on the other hand, one is interested in chemical reactivity or physical properties, a study of 'habit' may be valuable. In this case, however, the equidimensional particle (for example, cube or sphere) should be regarded as one end-point in the scale, as in Wadell's sphericity number. Wadell ${ }^{2}$ expresses the sphericity by the fraction $d_{n} / D_{n}$, where $d_{n}$ is the diameter of the sphere of the same volume as the particle and $D_{s}$ is the diameter of the circumscribing sphere. A slight modification of this test may quite easily be applied to rectangular parallelepipeds, and if the sides are $a \geqslant b \geqslant c$ the degree to which the particle approaches the equidimensional habit (the cube) is clearly $\sqrt[3]{a b c} / a$, the perfect cube being represented by unity. The cube possesses no relative elongation along its axes, and one mode of establishing a scale in which the cube has relative elongation of 0 is to define the elongation as $1-\sqrt[3]{a b c / a}$. Some may find it easier to comprehend these assessments if the values are all multiplied by 100 , so that one can speak of a cube as being 100 per cent equidimensional, and an infinitely long needle as being 100 per cent 'acicular'.

A more detailed classification of shapes by Zingg ${ }^{3}$ takes ratio into consideration in a somewhat similar manner to that used by Mr. Whittaker; but Zingg's method leads to the recognition of tendencies towards four habits: spherical (for he approaches it from the sedimentary petrographer's point of view), diskshaped, rod.like and bladed.

It should be borne in mind that with microscopic particles, the measurement of two dimensions may be all that is practicable, and shape assessments based on this limited data may be better than no data at all ${ }^{4,5}$.

\footnotetext{
British Potteries Research Association, Penkhull, Stoke-on-Trent.

1 Nature, 155, 331 (1945).

Wadell, H., Pan-Amer. Geol., 61, 187 (1934).

${ }^{3}$ Zingg, T., Schweiz. Min. W. Pet. Mitt., 15, 39 (1935).

'Wadell, H., J. Geol., 43, 250 (1935).

${ }^{5}$ Rittenhouse, G., J. Sed. Petrol., 13, 79 (1943).
}

Frank Smithson.
The coefficients proposed by Mr. E. J. W. Whittaker $^{1}$ for expressing shapes of rectangular parallelepipeda are not, in my opinion, quite satisfactory. The acicularity coefficient $\left(a c: b^{2}\right)$ does not really express true acicularity, because the term 'acicular' means 'needle-shaped' and is commonly applied to elongated crystals with a cross-section approaching a regular polygon. The term 'acicular' cannot, strictly speaking, be applied to elongated tabular crystals, which are usually referred to as 'laths', if short, and 'blades', if long. Again, the isoproportionality coefficient $(a: b$ or $b: c)$ does not express any significant shape, because it is based on two dimensions only.

Various coefficients can be devised for expressing the habit of crystals; but I think that the following coefficients are better suited to express the characteristic shape of a rectangular parallelepipedon $(a \geqslant b \geqslant c):$
1. Elongation $E=\frac{a^{2}-b^{2}}{b c}$.
2. Tabularity $T=\frac{a b^{2}-a c^{2}}{b c^{2}}$.

For a cube, both these coefficients are equal to zero.

King's College,

S. I. TomkenefF.

Newcastle-upon-Tyne.

${ }^{1}$ Nature, 155, 331 (1945).

Mr. S. I. TomkenefF's criticisms of the coefficients I have proposed for expressing particle shape are based on two points: the term 'acicularity' applied to the coefficient $a c: b^{2}$, and the use of $a: b$ or $b: c$ as the second shape parameter. With regard to the definition of acicularity, I would point out that my use of this term is an extension of its common meaning, so that it may be applied in a generalized sense to figures with three unequal principal dimensions. This extension is quite analogous to the extension of the terms positive and negative as applied to the indicatrix of biaxial crystals in crystal optics, these terms being derived from their use in describing the indicatrix of uniaxial crystals. Also, although my isoproportionality coefficient is not an explicit function of all three dimensions, it is not independent of any of their values owing to its definition as $a: b$, or $b: c$, whichever is the smaller, and it does, in combination with the acicularity coefficient, uniquely express any shape. Although there is a loss in mathematical rigour here, there is a great gain in simplicity.

Satisfactory coefficients for describing particle shape must satisfy the two mathematical criteria : (1) The coefficients must be homogeneous functions of $a, b$ and $c$. (2) They must be independent.

They should also satisfy, so far as possible, the following four conditions : (3) The coefficients applicable to a complex shape should analyse that shape into two variables which are readily separated subjectively in the impression given by the complex shape. (4) Their numerical values should vary along a series of shapes in accordance with subjective estimates of the changes occurring from one shape to another. (5) The coefficients should be as easily calculable as possible from the values of $a, b$ and $c$. (This is of considerable practical importance if a large number of particles is being measured.) 